\title{
Etnobotânica de espécies vegetais medicinais no tratamento de transtornos do sistema gastrointestinal
}

GOIS, M.A.F.1; LUCAS, F.C.A.1*; COSTA, J.C.M.1; MOURA, P.H.B. DE'; LOBATO, G. DE J.M.1

1 Universidade do Estado do Pará, Rua Djalma Dutra, s/n, CEP: 66.050-540, Belém-Pará. *Autor para correspondência: copaldoc@yahoo.com.br

\begin{abstract}
RESUMO: O estudo objetivou selecionar na comunidade Rio Urubueua de Fátima, AbaetetubaPará, as espécies vegetais utilizadas no tratamento de transtornos do sistema gastrointestinal, em determinado contexto de uso, associado a um conhecimento construído localmente. A pesquisa foi realizada com 35 informantes entre 28 e 93 anos, selecionados pelo método bola de neve. Os dados foram obtidos por observação participante e entrevistas semiestruturadas. Para a importância relativa das espécies vegetais, calculou-se a porcentagem de concordância quanto aos usos principais (CUP) e concordância quanto aos usos principais corrigida (CUPc). Os interlocutores indicaram várias receitas terapêuticas, e, destas, foram escolhidas as mais empregadas no tratamento da diarreia, por ser doença recorrente na comunidade. Foi investigado o potencial químico das plantas por meio de literatura científica e bancos de dados. "Boldo" e "Anoerá" apresentaram valor máximo de CUP (100\%), enquanto a "Hortelã" obteve maior CUPc (87,5\%). Das 79 espécies vegetais empregadas como medicinais, nove estão na lista do SUS. Os remédios são preparados exclusivamente sob a forma de chá, sendo a folha a parte da planta mais utilizada (65\%). Para o tratamento de diarreia, 12 espécies utilizadas pelos interlocutores têm compostos químicos comprovados por literatura especializada. A comunidade de Rio Urubueua de Fátima faz uso das plantas medicinais para curar doenças, apropriando-se de conhecimentos obtidos, na maioria dos relatos, de seus antepassados. Estes saberes tradicionais contribuem no conhecimento cultural da região e na pesquisa e desenvolvimento de novos fármacos.
\end{abstract}

Palavras-chave: Etnobotânica, medicina tradicional, plantas medicinais, Abaetetuba.

\begin{abstract}
Ethnobotany of medicinal species in the treatment of gastrointestinal system disorders. This study aimed to select the plants used to treat disorders of the gastrointestinal system, in the Rio Urubueua community of Fatima, Abaetetuba, and Pará for their particular context of use, combined with local knowledge. The survey was conducted with 35 informants between the ages of 28 and 93, selected using the snowball method.Data was obtained by participant observation, semi-structured interviews and by non-specific induction. To show the relative importance of plant species, we calculated the percentage of agreement on the main uses (CUP) and agreement with regards to the corrected main uses (cUPC). The interlocutors indicated various therapeutic recipes, and from these, those most used in the treatment of diarrhea were chosen, due to having recurrent cases of this disease in the community. The chemical potential of plants was investigated using scientific documents and databases. "Boldo" and "Anoerá" showed a maximum CUP value $(100 \%)$, while the "Hortelã" obtained a higher cUPC $(87.5 \%)$. Of the 79 plant species used in traditional medicine in this community, nine are used by the national healthcare service(SUS). The drugs are prepared only in the form of tea, with the leaves being the most widely used plant parts (65\%).For the treatment of diarrhea, the 12 species used by the interlocutors have chemical compositions that are supported by specialized literature. The Rio Urubueua community of Fatima makes use of medicinal plants to cure diseases, from knowledge obtained, in most cases, from their ancestors. This traditional knowledge contributes to the cultural knowledge of the region and to the research and development of new drugs.
\end{abstract}

Key words: Ethnobotany, traditional medicine, medicinal plants, Abaetetuba. 


\section{INTRODUÇÃO}

O interesse dos povos em relação ao meio ambiente, e em especial aos vegetais, data de milhares de anos (Leite et al., 2015). As primitivas civilizações notaram que existiam plantas que quando experimentadas no combate às doenças, revelaram o seu potencial curativo (Lima et al., 2007). Quando os primeiros europeus chegaram ao Brasil visualizaram de imediato grande quantidade de espécies medicinais empregadas por povos indígenas, e nessa troca de culturas que houve profundo envolvimento com a flora local e seu poder curativo, e os saberes trazidos da Europa e da África, principalmente, por escravos africanos (Amorozo, 2002).

Informações sobre plantas, pessoas e cultura, associado a um registro experimental de uso, com comprovado efeito biológico são analisadas e estudadas por disciplinas como a etnobotânica e a etnofarmacologia. Estas abordagens propiciam a seleção de espécies indicadas com base em saberes locais, focando a aplicação que fazem deles em seus sistemas de saúde e doença (Albuquerque \& Hanazaki, 2006). A partir disso, obtêm-se informações da etiologia da doença, medicamento, modo de administração, objetivos terapêuticos e outros detalhes que subsidiam o entendimento da cultura local (Etkin, 2001).

Plantas com propriedades curativas, ainda hoje, se constituem como principal alternativa para a cura de doenças em comunidades tradicionais por todo o mundo (Jesus et al., 2009). Para o tratamento de doenças gastrointestinais plantas como a ervacidreira (Lippia alba (Mill) N. E. Brown, Verbenaceae) que atua em perturbações digestivas, hepáticas, diarreicas e disenterias (Barbosa et al., 2006) e a goiaba (Psidium guajava L., Myrtaceae) que contém nas suas folhas propriedades antidiarreicas (Olajide et al., 1999; Lin et al., 2002) têm amplo emprego, principalmente em face à carência no atendimento à saúde pública ou acesso restrito à medicamentos.

No Brasil, apesar das diversas contribuições científicas quanto aos aspectos fitoquímicos e atividade biológica (Jorge et al., 2004; Duarte et al., 2004; Silva \& Faria, 2014), ainda são escassos os resultados para a descoberta de novos fármacos, pois muitas das plantas listadas com potencial terapêutico a partir do saber tradicional ainda não foram investigadas quanto à sua eficácia do ponto de vista farmacológico (Di Stasi, 2002).

Estudos etnobotânicos, como Germano et al. (2014) e Moura (2012), realizados na Comunidade ribeirinha de Rio Urubueua de Fátima, Abaetetuba-Pará, revelaram a aplicação de diversas plantas que tratam doenças diarreicas, verminoses e dores estomacais, sinalizando íntima relação entre os moradores e os recursos vegetais medicinais dentro do contexto sociocultural. Etnoespécies foram citadas, bem como quadros sintomáticos e as receitas empregadas para cada doença.

Com uma abordagem etnodirigida, este trabalho objetivou selecionar, na comunidade Rio Urubueua de Fátima, Abaetetuba-Pará, as espécies indispensáveis no tratamento de transtornos do sistema gastrointestinal, em determinado contexto de uso, associado a um conhecimento construído localmente, como forma de contribuir com a descoberta de novos fármacos de interesse médico ou farmacêutico. Para tanto, foram formuladas as seguintes questões: Quais são as espécies vegetais, formas de uso e partes da planta utilizadas no tratamento do sistema gastrointestinal? Quais são as receitas amplamente empregadas na comunidade para cura das doenças?

\section{MATERIAL E MÉTODOS}

\section{Autorização de acesso ao Patrimônio genético e conhecimentos tradicionais}

Antes de iniciar a pesquisa, foi realizada reunião junto à comunidade para apresentação do projeto e assinatura do Termo de Anuência Prévia (TAP). Posteriormente, foi solicitada autorização ao Conselho de Gestão do Patrimônio Genético (CGEN), Ministério do Meio Ambiente, que foi concedida na $104^{\circ}$ Reunião Ordinária, cujo processo $\left(n^{\circ} 02000.000586 / 2012-80\right)$ foi apreciado por meio da deliberação de autorização do CGEN n 148/2013.

\section{0 ambiente de estudo}

O município de Abaetetuba está localizado na mesorregião do nordeste paraense, com população estimada em 141.100 habitantes, que tem como atividades econômicas o comércio, pecuária, agricultura e extrativismo, especialmente de madeira, palmito e frutos de açaí e miriti (IBGE, 2010). A comunidade Rio Urubueua de Fátima (S 0137'92"; W 4858'42") está inserida em uma das 72 ilhas que também formam o município (Hiraoka \& Rodrigues, 1997). A vegetação é típica de ecossistemas de várzea, com predomínio de algumas espécies como o açaí (Euterpe oleracea Mart.), miriti (Mauritia flexuosa L.f.), mangueiro (Rhizophora racemosa G. Mey.), seringueira (Hevea brasiliensis (Willd. Ex A.Juss.) Müll.Arg.), aninga (Montrichardia linifera (Arruda) Schott) e a munguba (Pachira aquatica Aubl.) (Germano et al., 2014).

Em Rio Urubueua de Fátima não há saneamento básico ou coleta de lixo, o qual é queimado ou enterrado nos quintais (Germano et al., 2014). Existe um Posto de Saúde, de responsabilidade da Prefeitura de Abaetetuba-Pará, 
no qual há poucos medicamentos e a atenção dispensada à saúde dos moradores é insuficiente. Para os tratamentos mais especializados, os moradores se deslocam à Abaetetuba ou até a capital do estado, Belém. A pessoa responsável pelo posto é uma senhora de 74 anos, técnica de enfermagem, que realiza todos os atendimentos de rotina e emergência. Esta senhora é referência na comunidade pelo número de atendimentos bemsucedidos que realizou ao longo de mais de 30 anos de trabalho no local e por seu conhecimento com as plantas medicinais, as quais, associadas aos remédios farmacológicos, são utilizados pelos moradores.

\section{Coleta e Análise de dados}

Participaram da pesquisa 35 pessoas, dos gêneros masculino $(5,7 \%)$ e feminino $(94,3 \%)$, compreendendo idades entre 28 e 93 anos, sendo a faixa etária predominante de 50 a $59(n=12)$. Os interlocutores foram selecionados pelo método bola de neve (Bailey, 1982), com o apoio de um informante principal, que indicava outras pessoas conhecedoras de plantas medicinais. A amostragem foi do tipo não probabilística.

No período de julho de 2012 a julho de 2013, foram efetuadas quatro viagens à comunidade, cada uma com duração de uma semana. Os dados foram coletados a partir de técnicas de observação participante e entrevistas semiestruturadas (Albuquerque et al., 2010). Após as entrevistas, quando o interlocutor se disponibilizava, foram realizadas turnês guiadas (Albuquerque et al., 2010), que consistiam em visitas ao quintal florestal (área de mata mais distante da casa) ou no quintal doméstico (situado nos arredores da casa), para a coleta de plantas.

As amostras botânicas foram coletadas segundo Martins-da-Silva (2002) e identificadas por um parataxonomista no herbário (MG) do Museu Paraense Emílio Goeldi. Nomes científicos e autorias foram atualizados de acordo com a base online da Lista de Espécies da Flora do Brasil (Forzza et al., 2015) e, quando necessário, também foi consultado o banco de dados Missouri Botanical Garden (Tropicos, 2015). As exsicatas foram depositadas no Herbário da Universidade do Estado do Pará (MFS) e no Herbário do Museu Paraense Emílio Goeldi (MG).

A partir da análise de todas as espécies medicinais citadas nos formulários, foram filtradas apenas àquelas relacionadas ao tratamento das enfermidades do trato gastrintestinal. Para mostrar a importância relativa das plantas utilizadas na comunidade, calculou-se a porcentagem de concordância quanto aos usos principais (CUP) através da fórmula:
CUP $=\frac{n^{\circ} \text { de informantes que citaram usos principais } \times 100}{n^{\circ} \text { de informantes que referiram uso da espécie }}$

O valor de CUP encontrado foi multiplicado por um fator de correção (CUPc), que corresponde ao número de informantes que mencionou a espécie, dividido pelo número de informantes que mencionou a espécie mais citada, no caso:

$$
F C=\frac{n^{\circ} \text { de informantes que citaram a espécie }}{\frac{\text { nUPC }}{n^{\circ} \text { de informantes que referiram a espécie mais citada }}}
$$

A CUP, a CUPc e o FC foram calculados apenas para as espécies com quatro ou mais citações de usos para o tratamento de doenças relacionadas ao sistema gastrointestinal.

Das receitas terapêuticas empregadas nos distúrbios gastrointestinal, incluindo tanto as simples (preparadas com apenas uma espécie de planta) quanto às compostas (combinadas com uma ou mais espécies), foram selecionadas aquelas com concordância de usos entre os moradores, e agrupadas com base em critérios de semelhança de composição e uso (dosagens e modo de preparo). Ademais, foi feito levantamento de informações acerca da provável ação terapêutica das plantas nos distúrbios do trato gastrintestinal, com base no roteiro de cada receita, elaborada com base no etnoconhecimento local: 1 . Como as receitas para cada distúrbio são preparadas, com medidas e dosagens; 2. Partes da planta utilizadas; 3 . Forma de administração.

Para as doenças que obtiveram maior número de receitas terapêuticas foram investigados os constituintes químicos das plantas presentes nestas receitas. Para isso, pesquisaram-se dados químicos e farmacológicos publicados em documentos científicos e bancos de dados (Scinfinder, Scielo, Jstor, Biological Abstract e Medline), como forma de avaliar o entendimento da manutenção de uso das plantas ao longo do tempo em decorrência de um efeito farmacológico (potencial bioativo).

\section{RESULTADOS E DISCUSSÃO}

Os ribeirinhos de Rio Urubueua de Fátima Os ribeirinhos de Rio Urubueua de Fátima vivem em habitações localizadas às margens do rio ou dos furos (canal que conecta lagos, um rio a um lago ou um rio a ele mesmo, neste último caso, fora da planície aluvial), construídas em madeira e suspensas, que compõe o típico cenário das residências do estuário amazônico.

Aágua do rio é fonte de sobrevivência para

Rev. Bras. PI. Med., Campinas, v.18, n.2, p.547-557, 2016. 
a alimentação animal, com a pesca de mariscos, mas frequentemente de camarão, e peixes, além de se constituir como matéria prima elementar para as atividades cotidianas, pois é a água que se bebe, que prepara os alimentos, que se faz a higiene do corpo, lavagem de roupas, limpeza das casas e o preparo dos chás com as plantas medicinais. Esta água chega as moradias por meio de tubulações, e são coletadas em recipientes de plástico ou alumínio após passarem por telas de filtragem presas nas partes terminais dos tubos. A água consumida é desinfetada pela própria população com hipoclorito de sódio, distribuído pelos Agentes Comunitários de Saúde apenas para as famílias cadastradas na Secretaria de Saúde do Município.

\section{Os recursos vegetais e sua importância na comunidade}

Foram identificadas 32 espécies vegetais, pertencentes a 23 famílias botânicas, com destaque para Lamiaceae, Fabaceae e Asteraceae. (Tabela 1). O nome vernacular Hortelã (Mentha sp.) obteve maior número de citações, seguida pela Goiaba (Psidium guajava L.), Caxinguba (Ficus máxima Mill.), Açaí (Euterpe oleracea Mart.), Boldo (Vernonia condensata Baker) e Marupazinho (Eleutherine plicata (Sw.) Herb.).

Lamiaceae, Asteraceae e Fabaceae mostraram maior número de espécies (três a quatro), conforme a tabela 1. Resultados semelhantes foram encontrados por Alves \& Povh

TABELA 1. Espécies vegetais citadas pelos interlocutores na Comunidade Rio Urubueua de Fátima, AbaetetubaPará. C: Cultivada Es: Espontânea.

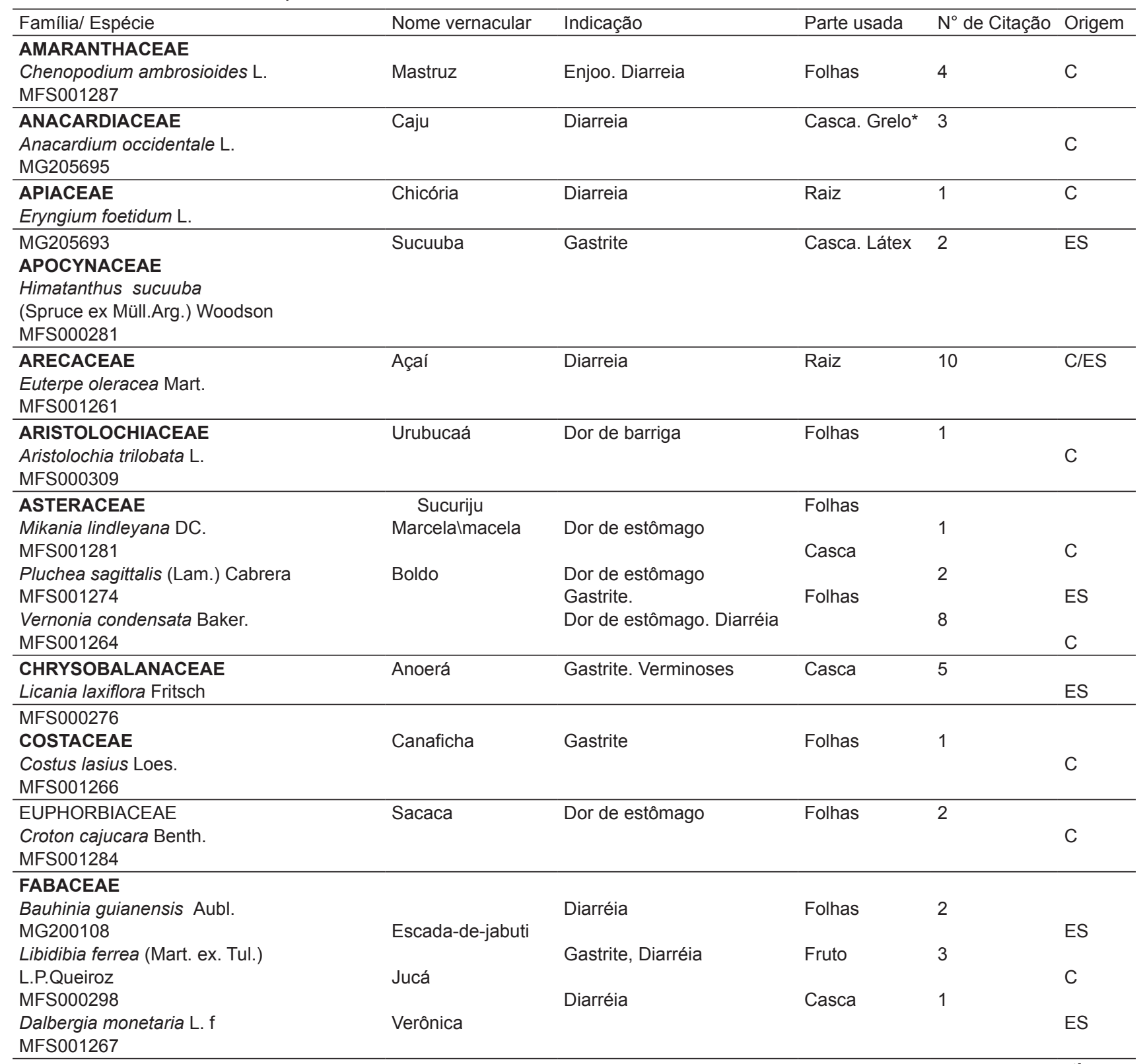


TABELA 1. Espécies vegetais citadas pelos interlocutores na Comunidade Rio Urubueua de Fátima, AbaetetubaPará. C: Cultivada Es: Espontânea.

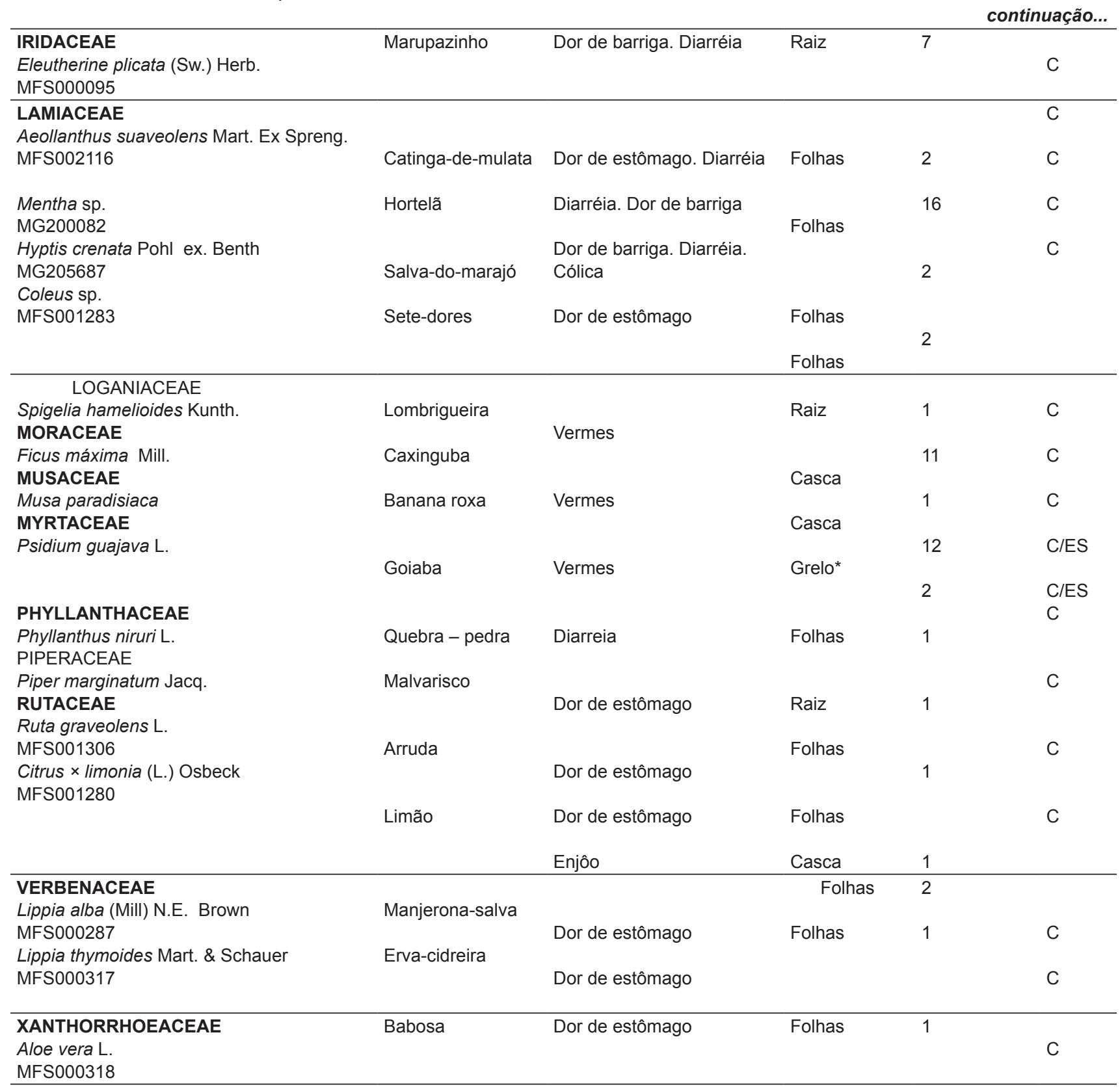

*Grelo: pontas dos ramos da árvore

(2013) na comunidade de Santa Rita, Itaituba-MG. De acordo com Judd et al. (2009) Lamiaceae possui representantes ricos em óleos essenciais, atribuindo às espécies propriedades aromáticas e medicinais. Asteraceae, considerada a maior família entre as Angiospermas, ressalta-se seu potencial medicinal e diversidade de substâncias químicas encontradas, principalmente, flavonoides, poliacetilenos, lactonas sesquiterpênicas, alcaloides, além de óleos voláteis e terpenoides (Cronquist, 1981 apud Tuler, 2011; Judd et al., 2009). Das espécies de Asteraceae em Rio Urubueua de Fátima, o boldo ( $V$. condensata) recebeu mais indicações para os agravos do sistema gastrointestinal.

Neste estudo, assim como em outros trabalhos (Cunha \& Bortolotto, 2011; Alves \& Povh, 2013), a folha é a parte da planta mais empregada nas receitas, totalizando $65 \%$, seguida da casca $(15 \%)$, raiz $(11 \%)$, galho $(5 \%)$, flores $(2 \%)$ e fruto (2\%). O uso frequente da folha, além de ser de fácil acesso, promove a conservação do recurso vegetal, pois não impede o desenvolvimento e a reprodução da planta, caso a retirada da parte aérea não seja excessiva (Belizário \& Silva, 2012).

No decorrer das entrevistas, ficou evidente a importância que algumas espécies têm quanto a 
sua eficácia, conforme observado nas verbalizações a seguir: "O mastruz e a hortelã são as espécies que considero com maior importância" (M.E, 43 anos); " Elixir paregórico e urubucaá são minhas plantas indispensáveis" (R.M, 37 anos). A hortelã, tão comum para tratar diferentes enfermidades, apresentou dezesseis citações de uso (Tabela 2), enquanto que a urubucaá resultou em uma citação. Os relatos evidenciam que, apesar da urubucaá ser citada apenas uma vez, é de suma importância na memória terapêutica da família que a citou. Ou seja, uma planta expressa valor, independente de seu uso ser amplo e popular.

A tabela 2 mostra a percentagem de concordância quanto aos usos principais (CUP), fator de correção (FC) e a CUP corrigida (CUPc) das espécies que obtiveram quatro ou mais citações de uso.

Anoerá e Boldo, ambas listadas para o tratamento de gastrite, demonstraram valor máximo de CUP. Contudo, revelaram valores baixos para CUPc, especialmente o anoerá, indicando baixo número de informantes que citou o uso principal para esta espécie, portanto, com menor concordância deste uso na comunidade. Na comunidade de Sisal, município de Catu, Bahia (Neto et al., 2014), o Boldo também obteve CUP máximo, salientando esta espécie como a mais relevante do acervo medicinal da comunidade, a qual é empregada pelos moradores para dor de estômago, gastrite e diarreia, podendo sugerir uma real efetividade no tratamento de doenças do sistema gastrointestinal.

Hortelã, Caxinguba, Goiaba e Açaí indicaram CUPc maiores que 50\%. O CUPc refere-se à fidelidade quanto a principal utilização da espécie citada pelos informantes, sinalizando que as espécies com um índice de concordância relativamente alto podem ser promissoras para estudos farmacológicos que possam corroborar a eficácia de suas propriedades terapêuticas
(Amorozo \& Gély, 1988; Vendruscolo \& Mentz, 2006).

\section{Receitas terapêuticas}

Foram indicadas 74 receitas terapêuticas para o trato gastrintestinal, sendo prioritárias nos seguintes distúrbios digestivos: diarreia, dor de barriga, gastrite, dor de estômago, vermes, amebas, úlceras e enjoos. Destas, 24 receitas são aplicadas somente para o tratamento de diarreia (Figura 1).

O elevado número de receitas a base de plantas medicinais destinadas a esse sistema sinaliza a gravidade com os problemas de saneamento básico em populações ribeirinhas amazônicas. Pesquisas etnodirigidas com populações em Salobrinho, Ilhéus, BA (Feijó et al., 2013) e em Sertão do Ribeirão, Florianópolis, SC (Giraldi \& Hanazaki, 2010), comprovaram que as plantas para os distúrbios gastrointestinais também estão relacionadas à precária qualidade da água.

As receitas citadas pelos interlocutores são preparadas, exclusivamente, na forma de chás, administrados oralmente, a partir de um preparado que pode incluir apenas uma planta (receita simples) ou, em $41,5 \%$ dos relatos, mais de uma (receita mista). Nas falas de um dos entrevistados, verificouse o uso combinado do marupazinho, hortelã e do açaí para o tratamento de diarreia: "pega dois galhinhos de hortelã, uma raiz vermelhinha do açaí e meia batata do marupazinho. Ferve tudo junto, coa num pano e depois dá" (A.C.D, 84, anos). O uso combinado de plantas foi retratado na Mata Atlântica (Pinto et al., 2006) e no Parque Estadual da Serra Furada, SC (Luca et al., 2014).

As 74 receitas listadas pelos interlocutores são amplamente empregadas e correspondem ao primeiro recurso de tratamento pelos moradores de Rio Urubueua de Fátima. Destas, 18 mostraram concordâncias no modo de preparo e a sua finalidade terapêutica (Tabela 3).

TABELA 2. Importância relativa, concordância quanto aos usos principais (CUP), fator de correção (FC), e concordância quanto aos usos principais corrigida (CUPc) das espécies vegetais catalogadas na comunidade Rio Urubueua de Fátima, Abaetetuba-Pará.

\begin{tabular}{ccccccc}
\hline $\begin{array}{c}\text { Nome } \\
\text { vernacular }\end{array}$ & $\begin{array}{c}\text { Informantes que } \\
\text { citaram o uso da } \\
\text { espécie }\end{array}$ & $\begin{array}{c}\text { Usos } \\
\text { principais }\end{array}$ & $\begin{array}{c}\text { Informantes que } \\
\text { citaram usos } \\
\text { principais }\end{array}$ & CUP & FC & CUPc (\%) \\
\hline Açaí & 10 & Diarreia & 9 & 90 & 0,6 & 54 \\
Anoerá & 5 & Vermes. Gastrite & 5 & 100 & 0,3 & 16,7 \\
Boldo & 8 & $\begin{array}{c}\text { Dor de estômago. } \\
\text { Gastrite }\end{array}$ & 8 & 100 & 0,6 & 44,4 \\
Caxinguba & 11 & Vermes & 11 & 84,6 & 0,7 & 61,1 \\
Goiaba & 12 & Diarreia & 10 & 83,3 & 0,7 & 58,3 \\
Hortelã & 16 & Diarreia.Vermes & 14 & 87,5 & 1 & 87,5 \\
Marupazinho & 7 & Dor de barriga. Diarréia & 5 & 71,4 & 0,4 & 28,5 \\
Mastruz & 6 & Vermes Gastrite & 5 & 83,3 & 0,3 & 27,8 \\
\hline
\end{tabular}

Rev. Bras. PI. Med., Campinas, v.18, n.2, p.547-557, 2016. 


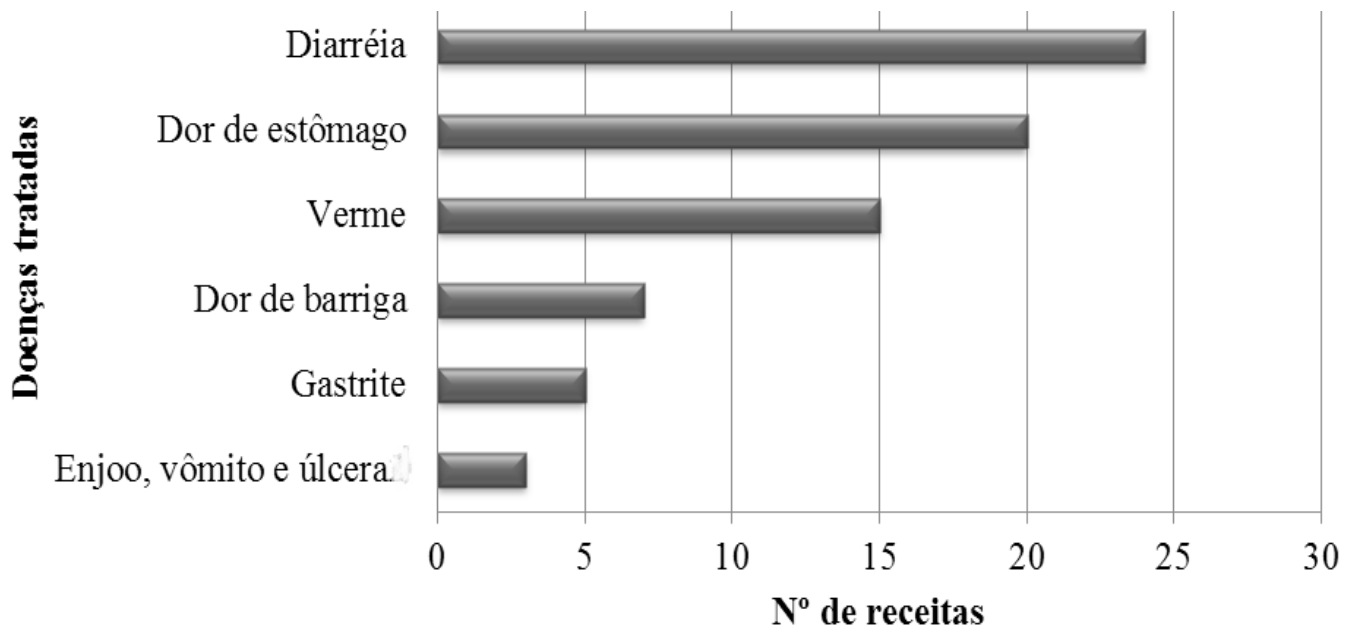

FIGURA 1. Receitas a base de plantas medicinais inventariadas em Rio Urubueua de Fátima para tratamento de doenças gastrointestinais.

No preparo das receitas os interlocutores relataram que frequentemente trocam plantas entre si. Essa prática é de grande importância, haja vista que muitas das espécies receitadas não são encontradas em todos os quintais. Esse comportamento também foi observado por Arnous, et al. (2005) no município de Datas, MG e por Neto et al. (2014) na Comunidade de Sisal, Catu, BA, evidenciando que há compartilhamento de plantas e saberes entre os moradores.

É importante salientar a representatividade e importância dessas receitas que são executadas com detalhes pelos conhecedores locais. São informações adquiridas por gerações pretéritas, ao longo de um tempo imemorial, conforme aponta a verbalização a seguir: "Aprendi com a minha mãe. Com a minha mãe, com a minha sogra também" (F.S., 47 anos).

\section{medicinais}

\section{O potencial químico das espécies}

As plantas na comunidade significam importante fonte de alimento e tratamento terapêutico para a família e amigos que chegam da cidade de Abaetetuba e de outras localidades do estado do Pará. A relativa valorização desse uso pode ser avaliada quando comparada à Relação Nacional de Plantas Medicinais de Interesse ao SUS (RENISUS), que lista 71 espécies aproveitadas como fitoterápicos no SUS ( Brasil, 2009). Desse total, nove fazem parte do presente estudo, como o barbatimão, que contém alcaloides, flavonoides, taninos, dentre outros, sendo esse último composto o constituinte majoritário, o qual agrega à espécie o valor medicinal (Panizza et al., 1988). Das outras oito plantas aqui encontradas, estão Caju (Anacardium occidentale L.), Hortelã (Mentha sp.), Boldo (Vernonia condensata Baker.), Goiaba (Psidium guajava L.), Marupazinho (Eleutherine plicata (Sw.) Herb.), Mastruz (Chenopodium ambrosioides L.), Jucá (Libidibia ferrea (Mart. ex. Tul.) e Arruda (Ruta graveolens L.).

Estudos fitoquímicos e farmacológicos são necessários para identificar os componentes e testar suas ações farmacológicas com base nos usos locais. Em Rio Urubueua de Fátima, 12 nomes vernaculares empregados apenas para o tratamento de diarreia encontram-se na Tabela 4, com seus compostos químicos comprovados por literatura especializada.

O açaí foi a terceira espécie mais citada nas receitas para o tratamento de diarreia e verminoses, o que também está em semelhança com os estudos de Jardim \& Medeiros (2006) para plantas oleaginosas do Estado do Pará, nos quais observaram que a raiz do açaí possui utilidade medicinal, sendo indicada para o tratamento de verminoses.

A indicação do caju para tratar verminoses e diarreia na comunidade está associada à sua atividade antimicrobiana (Silva et al., 2007). Estes autores sugeriram investigações aprofundadas, uma vez que o caju tem uso bastante difundido na cultura popular. Nos estudos de Periyanayagam et al. (2013) foi observado a presença de flavonoides e taninos, o que confere a esta espécie sua propriedade medicinal.

No presente estudo, verificou-se o amplo emprego da raiz do Marupazinho no combate à diarreia. Resultados semelhantes foram encontrados nos estudos etnofarmacêuticos de Barbosa \& Pinto (2001), na cidade de Igarapé-Miri, e em investigações etnobotânicas feitas por Jardim \& Medeiros (2006), no município de Santa Bárbara, ambos no estado do Pará, que ressaltaram o uso de Eleutherine plicata Herb. (marupazinho) no tratamento de diarreia, 
TABELA 3. Receitas semelhantes quanto à composição, indicação e modo de preparo, reportadas pelos moradores de Rio Urubueua de Fátima.

\begin{tabular}{|c|c|c|}
\hline ReceitasImodo de preparo & $\begin{array}{l}\text { Indicação } \\
\text { terapêutica }\end{array}$ & $\begin{array}{l}N^{\circ} \text { de Informantes que } \\
\text { fazem uso da receita }\end{array}$ \\
\hline $\begin{array}{l}\text { Receita } 1 \\
01 \text { a } 03 \text { dedos da casca da caxinguba. } 1 \text { a } 02 \text { copos de água. Ferve tudo } \\
\text { e depois coa. Tomar o chá por dois dias. }\end{array}$ & Vermes & 6 \\
\hline $\begin{array}{l}\text { Receita } 2 \\
01 \text { a } 02 \text { galhos de hortelã. } 1 \backslash 2 \text { a } 1 \text { batata de marupazinho. } 01 \text { copo de } \\
\text { água. Ferver tudo e tomar durante o dia }\end{array}$ & Diarréia & 2 \\
\hline $\begin{array}{l}\text { Receita } 3 \\
01 \text { galho de hortelã. } 01 \text { copo de água. Ferver os ingredientes por } 5 \mathrm{~min} \text { e } \\
\text { tomar durante as crises de diarreia. }\end{array}$ & Diarreia & 3 \\
\hline $\begin{array}{l}\text { Receita } 4 \\
01 \text { galho de hortelã. } 01 \text { copo de água. Ferver tudo junto. }\end{array}$ & Dor de barriga & 2 \\
\hline $\begin{array}{l}\text { Receita } \mathbf{5} \\
04 \text { a } 10 \text { folhas de boldo. } 01 \text { a } 02 \text { copos de água. Ferver tudo junto por } 5 \text { min, } \\
\text { coar e tomar quando sentir as dores. }\end{array}$ & $\begin{array}{l}\text { Dor de } \\
\text { estômago }\end{array}$ & 2 \\
\hline $\begin{array}{l}\text { Receita } 6 \\
1 \text { galho de mastruz. } 02 \text { copos de água. Ferver por } 3 \text { min, coar } \\
\text { e tomar em jejum. }\end{array}$ & $\begin{array}{l}\text { Gastrite e } \\
\text { vermes }\end{array}$ & 3 \\
\hline
\end{tabular}

Nota. 1 copo de água equivale a $200 \mathrm{~mL}$. 1 dedo da casca equivale a $10 \mathrm{~cm}$.

TABELA 4. Correlação de dados entre as espécies citadas pela comunidade Rio Urubueua de Fátima para o tratamento de diarreia e os compostos químicos associados segundo a literatura científica.

\begin{tabular}{|c|c|c|c|}
\hline Famílial Espécie & $\begin{array}{c}\text { Nome } \\
\text { vernacular }\end{array}$ & Classes de compostos & Referência \\
\hline AMARANTHACEAE & & Limonenos. & \\
\hline Chenopodium ambrosioides L. & Mastruz & Alfa terpenos & Cavalli et al., 2004 \\
\hline ANACARDIACEAE & & Taninos. Flavonóides. & Periyanayagam et al., \\
\hline Anacardium occidentale L. & Caju & Glicosídeos & 2013 \\
\hline $\begin{array}{c}\text { APIACEAE } \\
\text { Eryngium foetidum } \mathrm{L} .\end{array}$ & Chicória & $\begin{array}{l}\text { Flavonóides. } \\
\text { Triterpenóides }\end{array}$ & Wang et al., 2012 \\
\hline $\begin{array}{c}\text { ARECACEAE } \\
\text { Euterpe oleracea Mart. } \\
\text { ASTERACEAE }\end{array}$ & Açaí & Antocianinas & Jardim \& Medeiros, 2006 \\
\hline Vernonia condensata Baker. & Boldo & $\begin{array}{l}\text { Triterpenóides. } \\
\text { Esteróides }\end{array}$ & Lorenzi \& Matos, 2008 \\
\hline $\begin{array}{c}\text { FABACEAE } \\
\text { Bauhinia guianensis Aubl. } \\
\text { Dalbergia monetaria L. f. } \\
\text { LAMIACEAE }\end{array}$ & $\begin{array}{l}\text { Escada de jabuti } \\
\text { Verônica }\end{array}$ & $\begin{array}{l}\text { Esteróides; Flavonóides } \\
\text { Proantocianidinas }\end{array}$ & $\begin{array}{l}\text { Silva \& Filho, } 2002 \\
\quad \text { Cota, } 1999\end{array}$ \\
\hline $\begin{array}{c}\text { Aeollanthus suaveolens Mart. Ex } \\
\text { Spreng. } \\
\text { Mentha sp. } \\
\text { Hyptis crenata Pohl ex. Benth }\end{array}$ & $\begin{array}{l}\text { Catinga de } \\
\text { mulata } \\
\text { Hortelã } \\
\text { Salva do Marajó }\end{array}$ & $\begin{array}{l}\text { Tuiona. Terepenos. } \\
\text { Tanacetina } \\
\text { Mentol. Mentona. Cineol } \\
\text { Monoterpenos. } \\
\text { Sesquiterpenos }\end{array}$ & $\begin{array}{c}\text { Corrêa, } 1998 \\
\text { Silva, } 2001 \\
\text { Bravim, } 2008\end{array}$ \\
\hline $\begin{array}{c}\text { IRIDACEAE } \\
\text { Eleutherine plicata (Sw.) Herb. }\end{array}$ & Marupazinho & Taninos & $\begin{array}{l}\text { Lorenzi \& Matos, } \\
2002\end{array}$ \\
\hline $\begin{array}{c}\text { MYRTACEAE } \\
\text { Psidium guajava L. }\end{array}$ & Goiaba & $\begin{array}{l}\text { Taninos. Triterpenóides. } \\
\text { Flavonóides }\end{array}$ & Santos \& Mello, 2003 \\
\hline
\end{tabular}


possivelmente causada por ameba. Observou-se também nestes levantamentos que a raiz é a parte do vegetal aproveitada na preparação do chá para o tratamento desta patologia.

No presente estudo, o Boldo (Vernonia condensata) serve para o tratamento de gastrite, dor de estômago e diarreia. Para Costa \& Nascimento (2003) o Boldo é uma das plantas mais encontradas em levantamentos etnobotânicos medicinais no Brasil. Resultados de análises químicas registraram a presença de barbatusina, cariocal, além de triterpenoides e esteroides (Lorenzi \& Matos, 2008).

A Catinga-de-mulata (Aeollanthus suaveolens) e a Goiaba (Psidium guajava), empregada nos casos de diarreia, têm usos semelhantes nos trabalhos de Martins et al. (2005), Ilha do Combu, PA. Análises fitoquímicas comprovaram a presença de terpenos e tanacetina na Catinga-de-mulata (Corrêa, 1998), e para a goiaba, taninos, triterpenos e flavonoides (Santos \& Mello, 2003).

As espécies do gênero Mentha, conhecidas popularmente como hortelãs ou mentas, são amplamente administradas na medicina popular de vários países (Dorman et al., 2003). No presente estudo, a Hortelã (Mentha sp.) é a espécie mais citada nas receitas contra diarreia e dor de estômago. Na composição fitoquímica das espécies de Mentha encontra-se o mentol, mentona, mentofurano, acetato de mentila e pulegona (Aflatuni, 2005; Cappelo et al., 2007).

\section{CONCLUSÃO}

A comunidade de Rio Urubueua de Fátima utiliza com bastante frequência espécies vegetais como método para tratamento e cura de doenças gastrointestinais, com elevado emprego das plantas pertencentes às Asteraceae, Fabaceae e Lamiaceae. As receitas elaboradas fazem parte de conhecimentos apropriados não apenas pelos antepassados de cada família visitada, mas também por senhoras, moradoras da comunidade, consideradas especialistas em tratamentos a base de plantas. O papel dessas especialistas foi interpretado nesta pesquisa como mantenedor e propagador de repertórios que são validados por moradores de diferentes idades, incluindo primeiramente idosos, a seguir adultos e jovens.

A precariedade nos serviços públicos de saúde e no tratamento da água que provocam quadros frequentes de patologias gastrointestinais, estimularam: o uso recorrente de um número elevado de espécies vegetais; o cultivo destas na maioria dos quintais, sendo considerado como algo vital; e a elaboração e experimentação de numerosas receitas medicinais.

\section{REFERÊNCIA}

AFLATUNI, A. The yield and essential oil content of mint (Mentha ssp.) in northern ostrobothnia. 2005. 52p. Dissertação (Mestrado -Área de concentração em Biologia) - Faculdade de Ciências, Universidade de Oulu, Oulu,.

ALBUQUERQUE, U.P. HANAZAKI, N. As pesquisas etnodirigidas na descoberta de novos fármacos de interesse médico e farmacêutico: fragilidades e perspectivas Revista Brasileira de Farmacognosia, v. 16, 2006. p.678-689

ALBUQUERQUE, U.P.; LUCENA, R.F.P; CUNHA, L.V.F.C. Métodos e Técnicas na Pesquisa Etnobiológica e Etnoecológica. Vol 1. (Coleção Estudos e Avanços). NUPPEA: Recife, PE, Brasil. 2010. 560p.

AMOROZO, M.C.M; GÉLY, A.L. Uso de plantas medicinais por caboclos do Baixo Amazonas. Boletim do Museu Paraense Emilio Goeldi, v.4, n.1, p.47-131, 1988.

AMOROZO, M.C.M. Uso e Diversidade de Plantas Medicinais em Santo Antônio do Leverger MT, Brasil, Acta Botânica Brasílica, v.16, n.2, p.189-203, 2002.

ARNOUS, A.H.; et al., Plantas medicinais de uso caseiro: conhecimento popular e interesse por cultivo comunitário. Revista Espaço para a Saúde, v.6, n.2, p.1-6, 2005.

ALVES, G.S.P.; POVH, J.A. Estudo etnobotânico de plantas medicinais na comunidade de Santa Rita, Ituiutaba - MG. Revista Biotemas, v.26, n.3, p.231242, 2013.

BAILEY, K.D. Methods of Social Research. $4^{\text {a }}$ ed. The Free Press, New York, NY, USA. 1982. 439p.

BARBOSA, W.L.R.; PINTO, L.N. Levantamento etnofarmacêutico da fitoterapia tradicional de Igarapé-Miri. In: VII REUNIÃO DA SOCIEDADE BRASILEIRA PARA O PROGRESSO DA CIÊNCIA, V1. 2001 Manaus-AM.

BARBOSA, F.G. et al. Iridoid and phenylethanoid glycosides from Lippia alba. Biochemical Systematics and Ecology, v.34, p.819-821, 2006.

BELIZÁRIO, T.L.; SILVA, L.A. Abordagem etnobotânica no tratamento de parasitoses em comércios de fitoterápicos e numa comunidade rural em Uberlândia-MG. Enciclopédia Biosfera, v.8, n.15, p.1730-1739, 2012.

BRASIL. Ministério da saúde. Política Nacional de Plantas Medicinais e Fitoterápicos. Ministério da Saúde, Brasília, 2009. 136p. Disponível em: <portal.saude. gov.br/portal/ arquivos/pdf/RENISUS.pdf>. Acesso em 13 Jul. 2015.

BRAVIM, L.S. Avaliação da atividade antinociceptiva e anti-inflamatória do óleo essencial de Hyptis crenata (Pohl) ex Benth. 74 f. Dissertação (Programa de pós graduação em Ciências Farmacêuticas) Instituto de Ciências da Saúde, Universidade Federal do Pará, Belém.

CAPPELO, G.; et al., Peppermint oil (Mintoil) in the treatment of irritable bowel: a prospective double blind placebo controlled randomized trial. Digestive and Liver Disease, v.39, n.6, p.530-536, 2007.

CAVALLI, J.F.F.; et al., Combined analysis of the essential oil of Chenopodium ambrosioides by GC, GC-MS and 13C-NMR spectroscopy: quantitative determination of ascaridole, a heat-sensitive compound. Phytochemical 
Analysis, v.15, n.5, p.275-279, 2004.

CORRÊA, A.D. Plantas medicinais: do cultivo a terapêutica. $7^{\mathrm{a}}$ ed. Petrópolis, RJ: Vozes, 1998; 246p.

COSTA, M.C.C.D.; NASCIMENTO, S.C. Atividade citotóxica de Plectranthus barbatus Andr. (Lamiaceae). Acta Farmacêutica Bonaerense, v.22 n.2, p.155-158, 2003.

COTA, R.H.S dos. Ações anti ulcerogênicas do extrato aquoso liofilizado de Dalbergia monetaria L. (verônica). 1999. 90p. Dissertação (Pós Graduação em Ciências Biológicas) Universidade Estadual de Campinas, UNICAMP, Campinas, SP.

CUNHA, S.A. da; BORTOLOTTO, I.M. Etnobotânica de Plantas Medicinais no Assentamento Monjolinho, município de Anastácio, Mato Grosso do Sul, Brasil. Acta Botânica Brasilica, v.25, n.3, p.685-698, 2011.

DI STASI, L.C.; HIRUMA-LIMA C.A. Plantas Medicinais na Amazônia e na Mata Atlântica. $2^{\mathrm{a}}$ ed. UNESP: São Paulo. 2002. 608p.

DORMAN, H.J.D.; KOSAR, M.; KAHLOS, K.; HOLM, Y.; HILTUNEN, R. Antioxidant properties and composition of aqueous extracts from Mentha species, hybrids, varieties, and cultivars. Journal of Agricultural and Food Chemistry, v.51, n.16, p.4563-4569, 2003.

DUARTE, M.C.T.; et al., Atividade antimicrobiana de extratos hidroalcólicos de espécies da coleção de plantas medicinais CPQBAI UNICAMP. Revista Brasileira de Farmacognosia, v.14 p.06-08, 2004.

ETKIN, N.L. Perspectives in ethnopharmacology: forning a closer link betweenbioscience anda traditionalempiricalknowledge. J. ETHNOPHARMACOL, v.76, p.177-182, 2001.

FEIJÓ, E.V.R.S.; et al., Levantamento preliminar sobre plantas medicinais utilizadas no bairro Salobrinho no município de Ilhéus, Bahia. Revista Brasileira de Plantas Medicinais, v.15, n.4, p.595-604, 2013.

FORZZA, R. C., et al., Lista de Espécies da Flora do Brasil. Disponível em: <http://floradobrasil.jbrj.gov.br/>. Acesso em: 12 Jul. 2015.

GERMANO, C.M.; et al., Comunidades ribeirinhas e palmeiras no município de Abaetetuba, Pará, Brasil. Scientia Plena, v.10, n.11, p. 1-10, 2014.

GIRALDI, M; HANAZAKI, N. Uso e conhecimento tradicional de plantas medicinais no Sertão do Ribeirão, Florianópolis, SC, Brasil. Acta Botânica Brasílica, v.24, n.2, p.395-406, 2010.

HIRAOKA, M.; RODRIGUES, D.L. Porcos, palmeiras e ribeirinhos na várzea do estuário do Amazonas. In: FURTADO, L.G (Org.). Amazônia: desenvolvimento, sociobiodiversidade e qualidade de vida. Belém: UFPA/ NUMA, 1997. p.70-101.

IBGE. Instituto Brasileiro de Geografia e Estatística (IBGE). IBGE Cidades, 2010. Disponível em: http://www.ibge. gov.br/cidadesat/link.php?codigo=150010\&idtema=1. Acesso em 15 dez 2015.

JARDIM, M.A.G.; MEDEIROS, T.D.S. Plantas oleaginosas do Estado do Pará: composição florística e usos medicinais Revista Brasileira de Farmacognosia, v.87, n.4, p.124-127, 2006.

JESUS, N.Z.T.; et al.,. Levantamento etnobotânico de plantas popularmente utilizadas como antiúlceras e anti-inflamatórias pela comunidade de Pirizal, Nossa Senhora do Livramento - MT, Brasil. Revista Brasileira de Farmacognosia, v.19, n.1, p.130-139, 2009.

JORGE, R.M.; et al., Evaluation of antinociceptive, antiinflamatory and antiulcerogenio activities of Maytenusilicifolia. Journal Ethnopharmacol, v.94, p. 93-100, 2004.

JUDD, W.S.; CAMPBELL, C.S.; KELLOGG, E.A.; STEVENS, P.F.; DONOGHUE, M.J. Sistemática Vegetal: Um Enfoque Filogenético. 3. ed. Porto Alegre: Artmed, 2009. 632 p.

LEITE, I. A.; et al., A etnobotânica de Plantas Medicinais no Município de São José de Espinharas, Paraiba, Brasil. Biodiversidade, v. 14, n.1, p. 22-30, 2015.

LIMA, C.B.; et al., Uso de plantas medicinais pela população da zona urbana de Bandeirantes-PR. Revista Brasileira de Biociências, v. 5, supl.1, p. 600-602, 2007.

LIN, J.; PUCKREE, T.; MVELASE, T.P. Antidiarrhoealevaluationof some medicinal plantsusedby Zulu tradicional healers. Journal Ethnopharmacol, v.79, p. 53-56, 2002.

LORENZI, H.; MATTOS, F.J.A. Plantas Medicinais no Brasil: Nativas e Exóticas. $1^{a}$ ed. Nova Odessa: Instituto Plantarum, 2002. 512p.

LORENZI, H.; MATTOS, F.J.A. Plantas Medicinais no Brasil: Nativas e Exóticas. $2^{\mathrm{a}}$ ed. Nova Odessa: Instituto Plantarum, 2008. 560p.

LUCA, V.D.de; et al., A utilização de plantas medicinais no entorno do Parque Estadual da Serra Furada, Santa Catarina, Brasil; uma abordagem etnobotânica. Revista Brasileira de Biociências, v.12, n..2, p.59-65, 2014.

MARTINS, A.G.; et al., Levantamento etnobotânico de plantas medicinais, alimentares e tóxicas da Ilha do Combu, Município de Belém, Estado do Pará, Brasil. Revista Brasileira de Farmacognosia, v.86, n.1, p.2130, 2005.

MARTINS-DA-SILVA, R.C.V. Coleta e identificação de espécimes botânicos. $1^{\mathrm{a} e d}$. Embrapa Amazônia Oriental, Belém, PA, Brasil, 2002. 40p. Disponível em: <http://www.infoteca.cnptia.embrapa.br/handle/ doc/405766>. Acesso em 10 Jul. 2015.

MOURA, P.H.B.de. Estudo etnobotânico e caracterização dos constituintes minerais de chás medicinais utilizados pela comunidade Rio Urubueua de Fátima, Abaetetuba-PA, Brasil. 2012. 89p. Dissertação (Pós Graduação em Ciências Ambientais) Universidade do Estado do Pará, Belém-PA.

NETO, F.R.G.; et al., Estudo Etnobotânico de plantas medicinais utilizadas pela Comunidade do Sisal no município de Catu, Bahia, Brasil. Revista Brasileira de Plantas Medicinais, v.16, n.4, p.856-865, 2014.

NOLLA, D.; SEVERO, B.M.A. Plantas medicinais. 2 ed. Passo Fundo: UPF, 2005. 72p.

OLAJIDE,O.A.; AWE,S.O.; MAKINDE,J.M. Pharmacological studies on the leaf of Psidium guajava Fitoterapia, v.70, p.25-31, 1999.

PANIZZA, S.; et al.,. Stryphnodendron barbatimam (Vellozo) Martius: Teor em tanino na casca e sua propriedade cicatrizante. Revista Ciências Farmacêuticas, n.10, p.101-106, 1988.

PERIYANAYAGAM, K.; et al., Pharmacognostical and phytochemical studies on the leaves of Anacardium occidentale Linn. World Journal of Pharmaceutical Sciences, v.2, n.1., p.41-48, 2013.

PINTO, E.P.P.; et al., Conhecimento popular sobre plantas 
medicinais em comunidades rurais, em área de Mata Atlântica - Itacaré, BA. Acta Botânica Brasílica, v.20, n.4 p.751-752, 2006.

SANTOS, S.C., MELLO. J.C.P. Taninos. In: SIMÕES, C.M.O, SHENKEL E.P, GOSMANN, G., MELLO J.C.P, MENTZ, L.A, PETROVICK, P.R. Farmacognosia: da planta ao medicamento. 5. ed. Porto Alegre/ Florianópolis: Editora da UFRGS/ Editora da UFSC, 2003, p.615-656.

SILVA, A.R. Tudo sobre aromaterapia: como usá-la para melhorar sua saúde física, emocional e financeira. $2^{a}$ ed. São Paulo: Editora Roca Ltda, 2001. 624p.

SILVA K.L.; FILHO, V.C. Plantas do gênero Bauhinia: composição química e potencial farmacológico. Química Nova, v.25, n.3, p.449-454, 2002.

SILVA, J.G. et al. Atividade antimicrobiana do extrato de Anacardium occidentale L. em amostras multirresistentes de Staphylococcus aureus. Revista Brasileira de Farmacognosia, v.17, n.4, p.572-577, 2007.
SILVA, R.M, da; FARIA, M.T. Caracterização etnobotânica e histoquímica de plantas medicinais utilizadas pelos moradores do bairro Carrilho, Goianésia (GO). Enciclopédia Biosfera, v.10, n.19; p.2807-2829, 2014.

TROPICOS.org. Missouri Botanical Garden. Disponível em: http://www.tropicos.org. Acesso em 13 Jul. 2015.

TULER, A.C. Levantamento etnobotânico na Comunidade rural de São José da Figueira, Durandé, MG, Brasil. 2011. 57p. Graduação (Trabalho de Conclusão de Curso) Centro de Ciências Agrárias, Universidade Federal do Espírito Santo, Porto Alegre.

VENDRUSCOLO, G.S.; MENTZ, L.A. Levantamento etnobotânico das plantas utilizadas como medicinais por moradores do bairro Ponta Grossa, Porto Alegre, Rio Grande do Sul, Brasil. Acta Botânica Brasílica, v.61, n.1-2, p.83-103, 2006.

WANG, P.; et al., Phytochemical constituents and pharmacological activities of Eryngium L. (Apiaceae). Pharmaceutical Crops, n.3, p.99-120, 2012. 